\title{
Spin-dominated waveforms for unequal mass compact binaries
}

\author{
Márton Tápai ${ }^{\dagger}$, Zoltán Keresztes ${ }^{\ddagger}$, László Árpád Gergely* \\ Department of Theoretical Physics, University of Szeged, Tisza Lajos krt 84-86, Szeged 6720, Hungary \\ Department of Experimental Physics, University of Szeged, Dóm tér 9, Szeged 6720, Hungary \\ ${ }^{\dagger}$ E-mail: tapai@titan.physx.u-szeged.hu ${ }^{\star}$ E-mail: gergely@physx.u-szeged.hu ${ }^{\ddagger}$ E-mail: zkeresztes@titan.physx.u-szeged.hu
}

\begin{abstract}
We derive spin-dominated waveforms (SDW) for binary systems composed of spinning black holes with unequal masses (less than $1: 30$ ). Such systems could be formed by an astrophysical black hole with a smaller black hole or a neutron star companion; and typically arise for supermassive black hole encounters. SDW characterize the last stages of the inspiral, when the larger spin dominates over the orbital angular momentum (while the spin of the smaller companion can be neglected). They emerge as a double expansion in the post-Newtonian parameter $\varepsilon$ and the ratio $\xi$ of the orbital angular momentum and dominant spin. The SDW amplitudes are presented to $\left(\varepsilon^{3 / 2}, \xi\right)$ orders, while the phase of the gravitational waves to $\left(\varepsilon^{2}, \xi\right)$ orders (omitting the highest order mixed terms). To this accuracy the amplitude includes the (leading order) spin-orbit contributions, while the phase the (leading order) spin-orbit, self-spin and mass quadrupole-monopole contributions. While the SDW hold for any mass ratio smaller than $1: 30$, lower bounds for the mass ratios are derived from the best sensitivity frequency range expected for Advanced LIGO (giving 1 : 140), the Einstein Telescope $\left(7 \times 10^{-4}\right)$, the LAGRANGE $\left(7 \times 10^{-7}\right)$ and LISA missions $\left(7 \times 10^{-9}\right)$, respectively.
\end{abstract}

PACS numbers: 04.25.Nx, 04.30.Db, 04.80.Nn, 95.85.Sz

\section{INTRODUCTION}

The orbital evolution of the binary system forming compact objects is accompanied by emission of gravitational waves. The slight dissipative effect of the gravitational radiation allows for a long inspiral phase, followed by a short merger/plunge [1] and the relaxation of the newly formed object in a process called ringdown [2]. The inspiral dynamics can be accurately described by a post-Newtonian (PN) expansion in terms of a small (but ever increasing) parameter $\varepsilon=G m / c^{2} r \approx v^{2} / c^{2}$ (with $m=m_{1}+m_{2}$ the total mass, $r$ the orbital separation and $v$ the orbital velocity of the reduced mass particle $\left.\mu=m_{1} m_{2} / m\right)$.

The inspiral dynamics including the contributions of the spins and quadrupole moments was extensively discussed in Refs. 3]-[21]. The gravitational waveforms $h_{+}$ and $h_{\times}$characterizing the inspiral on circular orbits were given to $1 \mathrm{PN}$ order in Ref. [6], including the leading order spin-orbit contributions. In Ref. [14] the spinning waveforms were given to $1.5 \mathrm{PN}$ accuracy, and specified for the equal mass case by an expansion in the small angle $\iota$ (which will be denoted $\alpha$ in our formalism, and will not be small) span by the orbital angular momentum $\mathbf{L}_{N}$ and total angular momentum J. Spinning gravitational waveforms on generic orbits were also given in Ref. [22] to 1.5 PN order accuracy, and corrected in Ref. [23]. The multipole moments including spin effects to 2.5 PN order for gravitational wave amplitudes were calculated in Ref. [24].

The mass range of neutron stars is relatively narrow due to the upper bound on their masses represented by the Oppenheimer-Volkoff limit [25], 26]. Consequently the equal or nearly equal mass compact binary model stands as a good approximation during their inspiral.

The situation is however radically different for black hole binaries, and these are the compact objects, which have significant spin. There is no reason to believe that for astrophysical black hole binaries (with each black hole having a mass extending from a few solar masses, $\mathrm{M}_{\odot}$ to a few ten times of $\mathrm{M}_{\odot}$ ) the comparable mass case is more likely than any other mass ratio $\nu=m_{2} / m_{1}$. Moreover, for supermassive black hole binaries (with masses $\left.3 \times 10^{6}-3 \times 10^{9} \mathrm{M}_{\odot}\right)$ it has been shown that a mass ratio between 0.3 and 0.03 is typical [9], [10], [27] equal mass and extreme mass ratio encounters being disfavored. Low mass ratio and intermediate mass black hole binaries emitting gravitational waves are also believed to be sources for the LIGO detectors. However no signals were detected yet [28], [29], 30].With the advent of advanced LIGO [31], the planned space-born missions LISA-eLISA/NGO [32]-33] and LAGRANGE [34], also the third generation detectors, like the Einstein Telescope [35], the knowledge of waveforms from binaries with smaller mass ratio ranges becomes imperative. The small mass ratio parameter in these cases stands as a second small parameter, which modifies (and simplifies) some of the immediate results standing for the equal mass case. Indeed, it has been shown in Ref. 9], that the ratio of the spin magnitudes is

$$
\frac{S_{2}}{S_{1}}=\frac{\chi_{2}}{\chi_{1}} \nu^{2},
$$

where $\chi_{i} \in[0,1]$ are the dimensionless spin parameters. For rapidly spinning compact binaries and for small mass ratio the role of the second spin becomes negligible.

Moreover, the ratio of the dominant spin and magnitude of Newtonian orbital angular momentum is [9]

$$
\frac{S_{1}}{L_{N}} \approx \varepsilon^{1 / 2} \nu^{-1} \chi_{1}
$$

This relation shows that while for equal masses the inspiral is dominated by $L_{N}$, for small mass ratios this can 
change. Indeed, $S_{1}$ becomes dominant in the last stages of the inspiral for the unequal mass case $\nu<0.1$. (Obviously, in the test particle limit $S_{1}$ dominates throughout the inspiral, the orbital angular momentum of the test particle being negligible.)

In this paper we will focus on the situation when $\nu$ is small and we will derive the corresponding spinning waveforms to accuracy of order $\nu$. This immediately implies to disregard $S_{2}$ as of order $\nu^{2}$ compared to $S_{1} \cdot{ }^{1}$ As the gravitational wave amplitude increases drastically toward the end of the inspiral, we will concentrate on this regime. As has been shown in [9], [10], in this mass ratio range the end of the inspiral is characterized by $S_{1}$ dominating over $L_{N}$. Hence we call the corresponding gravitational waveforms as spin-dominated waveforms (SDW). We express this dominance by introducing a new small parameter

$$
\xi=\varepsilon^{-1 / 2} \nu
$$

assuming $\xi \leq 0.1$. As the PN parameter increases during the inspiral, this condition selects the last part of the inspiral starting from a given radius $r_{1}$. Hence the SDW are valid from $r_{1}$ to the end of the validity of the $\mathrm{PN}$ expansion. $^{2}$

We derive the SDW inspiral waveforms for circular orbits as a double expansion in the parameters $\varepsilon$ and $\xi$, with a linear accuracy in $\xi$ and $3 / 2$ orders in $\varepsilon$ (dropping however the $\varepsilon^{3 / 2} \xi$ terms). The introduction of the parameter $\xi$ leads to the natural neglection of the second spin, since $S_{i}=G / \mathrm{cm}_{i}^{2} \chi_{i}$ such that from Eq. (11) we find $S_{2}=\chi_{2} G / c m_{1}^{2} \nu^{2}=\chi_{2} G / \mathrm{cm}_{1}^{2} \varepsilon \xi^{2}$. Since the first terms containing spins in the amplitude are the $1 \mathrm{PN}$ order terms [6], the leading order terms containing $S_{2}$ would be shifted to $2 \mathrm{PN}$ orders in the amplitude, to be neglected in our approach (unless $\xi \gg 1$, which is not what we assume in this paper).

The structure of the paper is as follows. In Sec. [I] we analyze the limits of validity of the proposed SDW. In Sec. III and Appendix A we derive and present the SDW (gravitational wave amplitudes, with the spin-orbit corrections included) resulting from the double expansion. Here we employ that in the discussed parameter region the angle $\beta_{1}$ span by the dominant spin and total angular momentum is small, of order $\xi$. Therefore wherever possible, we express the angle $\alpha$ span by the total angular momentum and orbital angular momentum in terms of $\beta_{1}$ and perform the respective expansions to first order in $\xi$. Section IV] contains the expressions of the gravitational

\footnotetext{
${ }^{1}$ Gravitational waveforms with the inclusion of only one spin were previously investigated in Refs. [36], 37], to leading order in amplitude and 3.5 PN orders in the phase (the physical template family), however omitting the quadrupole-monopole and self-spin contributions, given later in Ref. [38].

2 The mass ratio $\nu$ will be replaced by $\varepsilon^{1 / 2} \xi$ in all expressions, with the exception of leading order contributions, where we keep $\nu$.
}

wave phase, expanded in terms of both $\varepsilon$ and $\xi$, with a linear accuracy in $\xi$ and second order in $\varepsilon$ (dropping however the $\varepsilon^{2} \xi$ terms). The phase then contains spin effects (the leading order spin-orbit and self-spin) and mass quadrupole-monopole effects, however spin-spin terms (of $\varepsilon^{5 / 2} \xi$ order) were dropped, as they would be of the same order in $\varepsilon$ as the PN correction of the spin-orbit terms [18], not included in our treatment.

Finally we present our concluding remarks in Sec. V]

\section{LIMITS OF VALIDITY}

During the inspiral, the PN parameter increases. Therefore the condition $\xi \leq \xi_{1}=0.1$ cannot be obeyed at distances larger than a certain $r_{1}$, which depends on the particular mass ratio. The value of the corresponding limiting $\mathrm{PN}$ parameter is $\varepsilon_{1}=G \mathrm{~m} / \mathrm{c}^{2} r_{1}=100 \nu^{2}$. Starting from these values of the separation and $\mathrm{PN}$ parameter, the approximation holds until the PN expansion breaks down. Levin, McWilliams, and Contreras argue in Ref. 39], that this should be at $\varepsilon_{2}=0.1$, and we adopt this upper limit. The argument goes as follows. At about $\varepsilon_{2}=0.1$ (well above the innermost marginally stable circular orbit) the PN expansion breaks down, as the 3.5 PN dissipation term (which is positive) becomes comparable to the $2.5 \mathrm{PN}$ order contribution (which is negative). Summing up, the regime we study lies between $\varepsilon_{1}$ and $\varepsilon_{2}$. With increasing mass ratio this interval shrinks, and it vanishes at $\nu_{\max }=0.0316 \approx 1: 32$.

For a specific gravitational wave detecting instrument, the lower frequency limit of the best sensitivity band and $\varepsilon_{2}$ determines the highest possible total mass of a binary source for the waves to be detected by the respective instrument. Kepler's third law gives a leading order estimate to the total mass $m$ of the system on circular orbit in terms of the gravitational wave frequency $f$ and $\mathrm{PN}$ parameter $\varepsilon$ as

$$
m=\frac{c^{3}}{\pi G} \varepsilon^{3 / 2} f^{-1} .
$$

The lower frequency bounds are $10 \mathrm{~Hz}$ for Advanced LIGO [31], $1 \mathrm{~Hz}$ for the Einstein Telescope [35], $10^{-3} \mathrm{~Hz}$ for LAGRANGE [34] and $10^{-5} \mathrm{~Hz}$ for LISA [32]. The respective values for $m_{\max }$ are thus $202 M_{\odot}$ (Advanced LIGO), $2020 M_{\odot}\left(\right.$ Einstein Telescope), $2 \times 10^{6} M_{\odot}$ (LAGRANGE) and $2 \times 10^{8} M_{\odot}$ (LISA).

Further, a lower limit $\nu_{\min }$ arises if we fix the smaller mass to be that of a neutron star. This leads to $\nu_{\min } \approx$ $0.007 \approx 1: 143$ for Advanced LIGO, $\nu_{\min } \approx 7 \times 10^{-4}$ for Einstein Telescope, $\nu_{\min } \approx 7 \times 10^{-7}$ for LAGRANGE and $\nu_{\min } \approx 7 \times 10^{-9}$ for LISA.

Next we estimate the time-interval spent by the binary evolving (due to gravitational radiation dissipation, considered to leading order) on quasicircular orbits from $\varepsilon_{1}$ to $\varepsilon_{2}$, as function of the mass ratio $\nu \in\left[\nu_{\min }, \nu_{\max }\right]$. For this we rewrite the leading order radiative orbital angular 

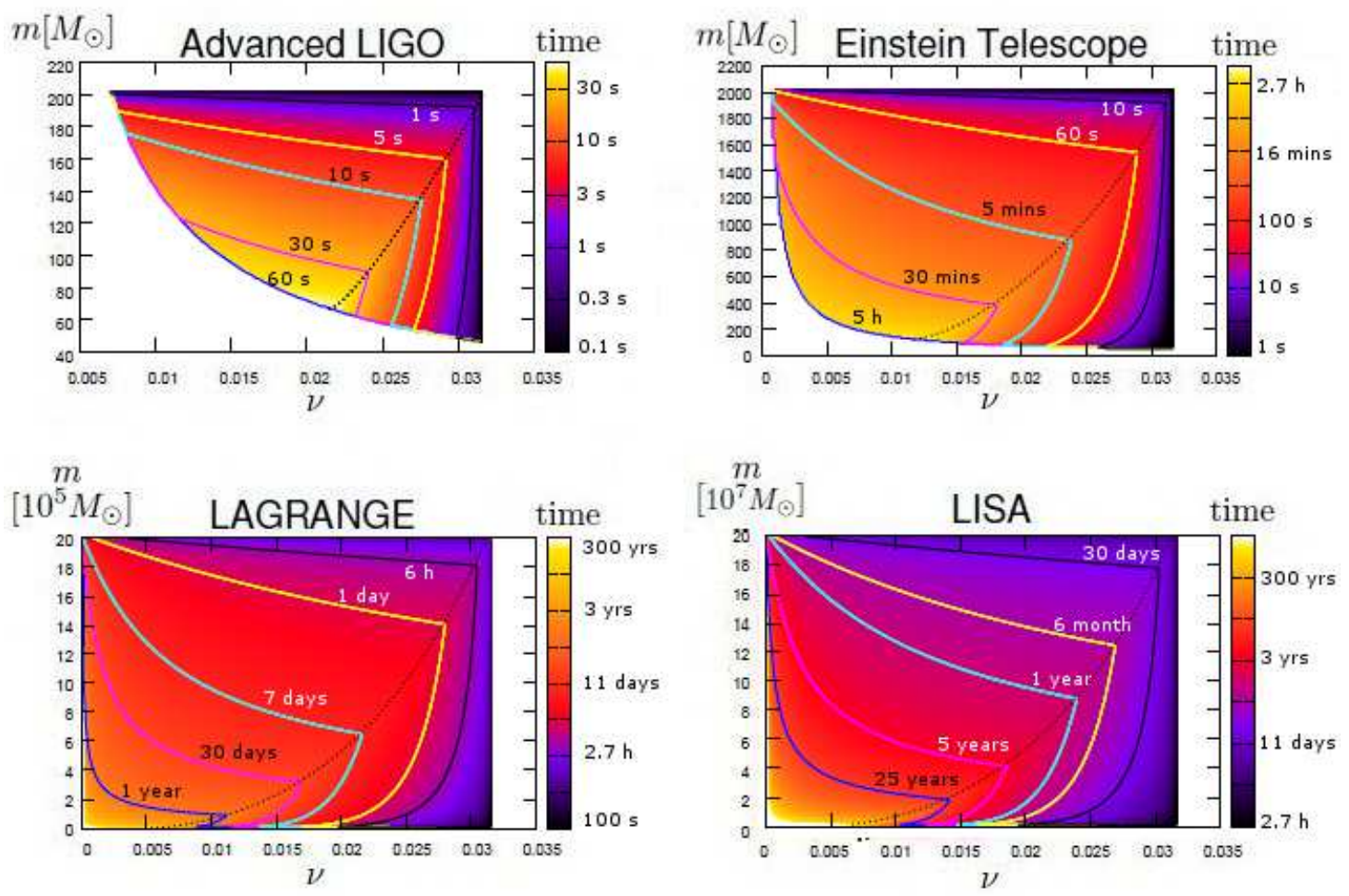

FIG. 1: (Color online) The color code and contour lines represent the time interval during which a system evolves from $\max \left(\varepsilon_{1}, \varepsilon_{f \min }\right)$ to $\varepsilon_{2}$ for Advanced LIGO (top left), Einstein Telescope (top right), LAGRANGE (bottom left), and LISA (bottom right) gravitational wave detectors, represented as function of the total mass $m$ and mass ratio $\nu$. The color code and contour lines are displayed logarithmically. The upper bound for the total mass emerges from the lower frequency limit of the best sensitivity range for each detector. The (total mass dependent) minimal mass ratio (visible on the top panels) arises by assuming the smaller mass to represent a neutron star with typical mass of $1.4 \mathrm{M}_{\odot}$. The maximal mass ratio (the cutoff on the right) is determined by the requirement, that $\varepsilon_{1}=100 \nu^{2} \leq 0.1$. In the larger part of the allowed parameter space (the region on the left of each dashed curve, representing $\left.\varepsilon_{1}=\varepsilon_{f \text { min }}\right)$ the gravitational waves entering into the best sensitivity range of the respective detectors are described by SDW. In the regions on the right of the dashed curves however SDW can not be applied from the lower frequency bound of the best sensitivity range, hence the time intervals are shorter.

frequency evolution given in Ref. [38] as

$$
\frac{(1+\nu)^{2}}{\nu} \omega^{-8 / 3}=\frac{2^{8}(G m)^{5 / 3}}{5 c^{5}}\left(t_{c}-t\right),
$$

(where $t_{c}-t$ is the time left until the final coalescence). Rewriting Eq. (44) as

$$
\varepsilon=\frac{(G m \omega)^{2 / 3}}{c^{2}}
$$

where $\omega=\pi f$, we obtain

$$
\frac{(1+\nu)^{2}}{\nu} \varepsilon^{-4}=\frac{2^{8} c^{3}}{5 G m}\left(t_{c}-t\right) .
$$

Then the time elapsed during the evolution from $\varepsilon_{1}$ to $\varepsilon_{2}$ can be calculated as

$$
\Delta t=\frac{5 G m}{2^{8} c^{3}} \frac{(1+\nu)^{2}}{\nu}\left(\varepsilon_{1}^{-4}-\varepsilon_{2}^{-4}\right) .
$$

Thinking of a specific instrument we can investigate the time necessary for the evolution starting from the lower frequency range $\left(f_{\min }\right)$ of a given detector (when this belongs to a value $\varepsilon_{f \text { min }}>\varepsilon_{1}$ ) until $\varepsilon_{2}$. On Fig. 1 we represent this time interval necessary for the evolution from $\max \left(\varepsilon_{1}, \varepsilon_{f}\right.$ min $)$ to $\varepsilon_{2}$ for the enlisted detectors (Advanced LIGO, Einstein Telescope, LAGRANGE, and LISA).

\section{SPIN-DOMINATED GRAVITATIONAL WAVEFORMS}

\section{A. Gravitational waveforms to $1.5 \mathrm{PN}$ order}

Gravitational waveforms including spin-orbit effects were previously calculated in Ref. [6] to $1.5 \mathrm{PN}$ order for nearly circular orbits, based on the radiative multipole moments of 1.5 PN order accuracy, and reproduced 
(with some misprints corrected) in Ref. [14] as

$$
\begin{aligned}
h^{i j}= & \frac{2 G \mu}{c^{4} D}\left(\frac{G m}{r}\right)\left[Q_{c}^{i j}\right. \\
& +P^{0.5} Q_{c}^{i j} \varepsilon^{1 / 2}+P Q_{c}^{i j} \varepsilon \\
& \left.+\left(P^{1.5} Q_{c}^{i j}+P^{1.5} Q_{\text {Tail }}^{i j}\right) \varepsilon^{3 / 2}\right]_{T T},
\end{aligned}
$$

where TT denotes the transverse trace-free projection into the plane orthogonal to the direction $\hat{\mathbf{N}}$ pointing from the source to the observer (the line of sight), and

$$
\begin{aligned}
& Q_{c}^{i j}=2\left[\lambda^{i} \lambda^{j}-\hat{r}^{i} \hat{r}^{j}\right], \\
& P^{0.5} Q_{c}^{i j}=\frac{1-\nu}{1+\nu}\left\{6(\hat{\mathbf{N}} \cdot \hat{\mathbf{r}}) \hat{r}^{(i} \lambda^{j)}\right. \\
& \left.+(\hat{\mathbf{N}} \cdot \hat{\boldsymbol{\lambda}})\left[\hat{r}^{i} \hat{r}^{j}-2 \lambda^{i} \lambda^{j}\right]\right\}, \\
& P Q_{c}^{i j}=\frac{2}{3}(1-3 \eta)\left\{(\hat{\mathbf{N}} \cdot \hat{\mathbf{r}})^{2}\left[5 \hat{r}^{i} \hat{r}^{j}-7 \lambda^{i} \lambda^{j}\right]\right. \\
& -16(\hat{\mathbf{N}} \cdot \hat{\mathbf{n}})(\hat{\mathbf{N}} \cdot \hat{\boldsymbol{\lambda}}) \hat{r}^{(i} \lambda^{j)} \\
& \left.+(\hat{\mathbf{N}} \cdot \hat{\boldsymbol{\lambda}})^{2}\left[3 \lambda^{i} \lambda^{j}-\hat{r}^{i} \hat{r}^{j}\right]\right\} \\
& +\frac{1}{3}(19-3 \eta)\left(\hat{r}^{i} \hat{r}^{j}-\lambda^{i} \lambda^{j}\right) \\
& +\frac{2 c}{G m^{2}} \frac{1+\nu}{1-\nu} \hat{r}^{(i}((\boldsymbol{\sigma}-\mathbf{S}) \times \hat{\mathbf{N}})^{j)}, \\
& P^{1.5} Q_{c}^{i j}=\frac{1-\nu}{1+\nu}\left\{( 1 - 2 \eta ) \left[\frac{1}{2}(\hat{\mathbf{N}} \cdot \hat{\boldsymbol{\lambda}})^{3}\left(\hat{r}^{i} \hat{r}^{j}-4 \lambda^{i} \lambda^{j}\right)\right.\right. \\
& +\frac{1}{4}(\hat{\mathbf{N}} \cdot \hat{\mathbf{r}})^{2}(\hat{\mathbf{N}} \cdot \hat{\boldsymbol{\lambda}})\left(58 \lambda^{i} \lambda^{j}-37 \hat{r}^{i} \hat{r}^{j}\right) \\
& \left.-\frac{65}{6}(\hat{\mathbf{N}} \cdot \hat{\mathbf{r}})^{3} \hat{r}^{(i} \lambda^{j)}+15(\hat{\mathbf{N}} \cdot \hat{\mathbf{r}})(\hat{\mathbf{N}} \cdot \hat{\boldsymbol{\lambda}})^{2} \hat{r}^{(i} \lambda^{j)}\right] \\
& -(\hat{\mathbf{N}} \cdot \hat{\boldsymbol{\lambda}})\left[\frac{1}{12}(101-12 \eta) \hat{r}^{i} \hat{r}^{j}-\frac{1}{2}(19-4 \eta) \lambda^{i} \lambda^{j}\right] \\
& \left.-\frac{1}{6}(149-36 \eta)(\hat{\mathbf{N}} \cdot \hat{\mathbf{r}}) \hat{r}^{(i} \lambda^{j)}\right\} \\
& -\frac{2 c}{m^{2} G}\left\{\lambda^{i} \lambda^{j}\left[\hat{\mathbf{L}}_{\mathbf{N}} \cdot(2 \mathbf{S}+3 \boldsymbol{\sigma})\right]\right. \\
& -6 \hat{r}^{i} \hat{r}^{j}\left[\hat{\mathbf{L}}_{\mathbf{N}} \cdot(\mathbf{S}+\boldsymbol{\sigma})\right] \\
& +2 \lambda^{(i}[\hat{\mathbf{r}} \times(\boldsymbol{\sigma})]^{j)}+\hat{r}^{(i}[\hat{\boldsymbol{\lambda}} \times(4 \mathbf{S}+5 \boldsymbol{\sigma})]^{j)} \\
& +2(\hat{\mathbf{N}} \cdot \hat{\boldsymbol{\lambda}})[(\boldsymbol{\sigma}) \times \hat{\mathbf{N}}]^{(i} \hat{r}^{j)} \\
& \left.+2(\hat{\mathbf{N}} \cdot \hat{\mathbf{r}})[(\boldsymbol{\sigma}) \times \hat{\mathbf{N}}]^{(i} \lambda^{j)}\right\},
\end{aligned}
$$

$$
\begin{aligned}
P^{1.5} Q_{\text {Tail }}^{i j}= & 4\left[\pi\left(\lambda^{i} \lambda^{j}-\hat{r}^{i} \hat{r}^{j}\right)\right. \\
& \left.+4 \ln \left(\frac{\omega}{\omega_{0}}\right) \hat{r}^{(i} \lambda^{j)}\right] .
\end{aligned}
$$

Here $r^{i}$ and $\lambda^{i}$ are the components of the separation vector and of $\boldsymbol{\lambda}=\mathbf{L}_{\mathbf{N}} \times \mathbf{r}=\mathbf{v} / r \omega$, the parameter $\eta=$ $\mu / m=\nu /(1+\nu)^{2}$ is the symmetric mass ratio, and we introduced the spin combinations $\mathbf{S}=\mathbf{S}_{1}+\mathbf{S}_{2}$ and $\boldsymbol{\sigma}=\nu \mathbf{S}_{1}+\nu^{-1} \mathbf{S}_{2}$. The last term, $P^{1.5} Q_{\text {Tail }}^{i j}$ arising from the contributions of the gravitational wave tails was first presented in this form in Ref. [14]. The parameter $\omega_{0}$ is an arbitrary constant frequency scale [40]. Unit vectors carry a hat.

The two gravitational wave polarization states $h_{+}$and $h_{\times}$emerge as the linear combinations

$$
\begin{aligned}
& h_{+}=\frac{1}{2}\left(\hat{\mathbf{x}}_{i} \hat{\mathbf{x}}_{j}-\hat{\mathbf{y}}_{i} \hat{\mathbf{y}}_{j}\right) h^{i j}, \\
& h_{\times}=\frac{1}{2}\left(\hat{\mathbf{x}}_{i} \hat{\mathbf{y}}_{j}+\hat{\mathbf{x}}_{j} \hat{\mathbf{y}}_{i}\right) h^{i j} .
\end{aligned}
$$

of the $h^{i j}$ tensor components perpendicular to $\hat{\mathbf{N}}[\underline{6}$, with $\hat{\mathbf{y}}=\mathbf{J} \times \hat{\mathbf{N}} /|\mathbf{J} \times \hat{\mathbf{N}}|$ and $\hat{\mathbf{x}}=\hat{\mathbf{y}} \times \hat{\mathbf{N}}^{3}$.

\section{B. The spin-dominated regime}

The total angular momentum $\mathbf{J}=\mathbf{L}_{\mathbf{N}}+\mathbf{L}_{\mathbf{P N}}+\mathbf{L}_{\mathbf{S O}}+$ $\mathbf{S}_{\mathbf{1}}+\mathbf{S}_{\mathbf{2}}$ is conserved to $2 \mathrm{PN}$ accuracy [5] . Here $\mathbf{L}_{\mathbf{P N}}=$ $\epsilon_{P N} \mathbf{L}_{\mathbf{N}}$ and $\mathbf{L}_{\mathbf{S O}}$ are the $\mathrm{PN}$ and spin-orbit corrections in the orbital angular momentum vector. Starting from their expressions Eq. (39) in Ref. 20] and Eq. (B23) in Ref.[21], and the definition of the PN parameter $\varepsilon$ we find

$$
\begin{aligned}
\frac{L_{P N}}{L_{N}}= & \epsilon_{P N} \approx \varepsilon\left[\frac{7}{2}-\frac{1}{2} \frac{\xi \varepsilon^{1 / 2}}{\left(1+\xi \varepsilon^{1 / 2}\right)^{2}}\right] \\
\frac{L_{S O}}{L_{N}} \approx & \frac{\xi^{2} \varepsilon^{5 / 2}}{4\left(1+\xi \varepsilon^{1 / 2}\right)^{4}}\left[4 \xi^{-1} \varepsilon^{-1 / 2} \chi_{1}\right. \\
& \left.+3\left(\chi_{1}+\chi_{2}\right)+4 \xi \varepsilon^{1 / 2} \chi_{2}\right] .
\end{aligned}
$$

Expanding these to linear order in $\xi$, we have

$$
\begin{aligned}
\frac{L_{P N}}{L_{N}} & =\mathcal{O}\left(\varepsilon, \varepsilon^{3 / 2} \xi\right) \\
\frac{L_{S O}}{L_{N}} & =\mathcal{O}\left(\varepsilon^{2} \xi \chi_{1}\right) .
\end{aligned}
$$

3 We mention that the polarization vectors $\hat{\mathbf{x}}$ and $\hat{\mathbf{y}}$ are rotated by $\pi / 2$ in Ref. [14], resulting a global sign difference in the definition of $h_{+}$and $h_{\times}$. 
Keeping the terms to $\varepsilon, \varepsilon \xi, \varepsilon^{3 / 2}$ orders only in the above set of equations, $\mathbf{J}$ becomes

$$
\mathbf{J}=\left(1+\frac{7}{2} \varepsilon\right) \mathbf{L}_{\mathbf{N}}+\mathbf{S}_{1}
$$

Therefore to the accuracy we are interested in, both the second spin and the spin-orbit contribution to the orbital angular momentum could be dropped. Then in the triangle with sides $S_{1},(1+7 \varepsilon / 2) L_{N}$ and $J$, containing the $\operatorname{angles} \alpha=\arccos \left(\hat{\mathbf{J}} \cdot \hat{\mathbf{L}}_{\mathbf{N}}\right)$ and $\beta_{1}=\arccos \left(\hat{\mathbf{J}} \cdot \hat{\mathbf{S}}_{\mathbf{1}}\right)$ the sine theorem gives

$$
\begin{aligned}
\sin \beta_{1} & =\left(1+\frac{7}{2} \varepsilon\right) \frac{L_{N}}{S_{1}} \sin \alpha \\
& =\left(1+\frac{7}{2} \varepsilon\right) \frac{\xi}{\chi_{1}} \sin \alpha .
\end{aligned}
$$

As $\alpha$ has no preferred value and $\xi$ is small, we conclude that $\beta_{1}$ is of order $\xi$. (From the assumption $\xi<0.1$ it follows that $\sin \beta_{1} \lesssim 0.1$.) Thus any trigonometric function of $\beta_{1}$ can be approximated by its Taylor expanded form, to first order accuracy (dropping $\xi^{2} \leq 0.01$ terms and higher).

It was shown in Ref. [9], that the angle $\kappa_{1}=\alpha+\beta_{1}$ stays constant during the inspiral, when the smaller spin is negligible. Therefore we replace $\alpha$ everywhere by $\kappa_{1}-$ $\beta_{1}$ and then expand to first order in $\beta_{1}$. We will show in the next subsection that the double expansion of $h_{+}$and $h_{\times}$in $\varepsilon$ and $\xi$ leads to the following structure of terms: $1, \beta_{1}, \varepsilon^{1 / 2}, \varepsilon^{1 / 2} \beta_{1}, \varepsilon, \varepsilon \xi, \varepsilon^{3 / 2}$. (We note here that the leading order contribution from the smaller spin $S_{2}$ would appear only at $\varepsilon^{2} \xi^{2}$ orders.) Since $\varepsilon$ increases during the inspiral, the terms $\beta_{1}, \varepsilon^{1 / 2} \beta_{1}, \varepsilon \xi$, all kept, increase as well, however the $\varepsilon^{2}$ term, discarded, increases at an even faster rate. Therefore when either of the enlisted terms becomes comparable to the $\varepsilon^{2}$ contribution, we have to drop it as well. Figure 2 shows the terms to be kept in the parameter space $(\varepsilon, \nu)$.

\section{SDW inspiral waveforms}

We give the gravitational wave polarizations (15) and (16) in the source coordinate system defined as follows. The $z_{S}$-axis lies along the direction of $\mathbf{J}$, and the $x_{S^{-}}$ axis is assigned by the projection of $\hat{\mathbf{N}}$ perpendicular to $\mathbf{J}$. The polar angle of $\hat{\mathbf{N}}$ will be denoted by $\theta$. In this coordinate system the components of the vectors $\mathbf{r}, \boldsymbol{\lambda}$, and $\mathbf{S}_{1}$ are

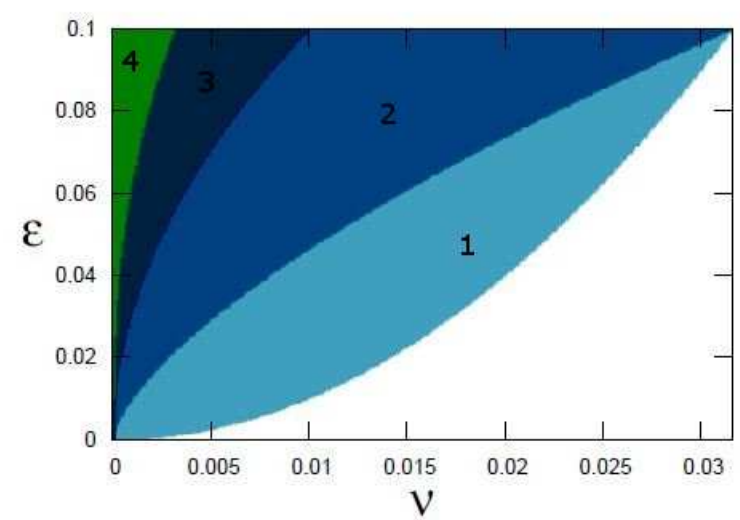

FIG. 2: (Color online) The figure represents the parameter ranges $\nu$ and $\varepsilon$ for which all terms given in the waveform are larger than the pure $2 \mathrm{PN}\left(\varepsilon^{2}\right)$ contributions (area 1$)$. In the parameter range 2 the contributions $\varepsilon \xi, \varepsilon \beta_{1}$ are becoming comparable with the pure $2 \mathrm{PN}$ terms, falling outside of the limits of validity of our approach, hence they can be neglected. In the parameter range 3 all mixed terms in the double expansion become negligibly small, while in the parameter range 4 all terms containing $\xi$ and $\beta_{1}$ can be dropped. In the white area, the condition $\xi \leq 0.1$ doesn't hold during the inspiral.

$$
\begin{aligned}
\frac{\mathbf{r}_{x}}{r}= & -\sin \left(\frac{3 \pi}{2}-\phi_{n}\right) \cos \phi \\
& -\cos \alpha \cos \left(\frac{3 \pi}{2}-\phi_{n}\right) \sin \phi \\
\frac{\mathbf{r}_{y}}{r}= & \cos \left(\frac{3 \pi}{2}-\phi_{n}\right) \cos \phi \\
& -\cos \alpha \sin \left(\frac{3 \pi}{2}-\phi_{n}\right) \sin \phi \\
\frac{\mathbf{r}_{z}}{r}= & \sin \alpha \sin \phi \\
\boldsymbol{\lambda}_{x}= & \sin \left(\frac{3 \pi}{2}-\phi_{n}\right) \sin \phi \\
& -\cos \alpha \cos \left(\frac{3 \pi}{2}-\phi_{n}\right) \cos \phi \\
\lambda_{y}= & -\cos \left(\frac{3 \pi}{2}-\phi_{n}\right) \sin \phi \\
& -\cos \alpha \sin \left(\frac{3 \pi}{2}-\phi_{n}\right) \cos \phi \\
\boldsymbol{\lambda}_{y}= & \sin \alpha \cos \phi \\
S_{1 x}= & \frac{G}{c} m^{2} \eta \nu^{-1} \chi_{1} \sin \beta_{1} \cos \left(\phi_{1}-\phi_{n}\right), \\
S_{1 y}= & \frac{G}{c} m^{2} \eta \nu^{-1} \chi_{1} \sin \beta_{1} \sin \left(\phi_{1}-\phi_{n}\right) \\
S_{1 z}= & \frac{G}{c} m^{2} \eta \nu^{-1} \chi_{1} \cos \beta_{1}
\end{aligned}
$$


Here the angle $\phi_{1}$ is the azimuthal angle of the dominant spin. $\phi_{n}$ is defined as the angle between the intersection of the planes perpendicular to $\mathbf{J}$ and $\mathbf{L}_{\mathbf{N}}$ and an arbitrary inertial axis taken in the plane perpendicular to $\mathbf{J}$, and $\phi$ is the orbital phase. These angles are shown on Figs. 1 and 2 of Ref. [20]. The time evolution of these angles was given in Ref. [21].

We substitute $\mathbf{r}, \boldsymbol{\lambda}$, and $\mathbf{S}_{1}$ from Eqs. (22)-(24) into the expression of $h_{+}$and $h_{\times}$given by Eqs. (15) and (16). The 1.5 PN tail contribution contains terms with $\ln \left(\omega / \omega_{0}\right)$, which can be absorbed into the leading order term by redefining the phase $\phi$ as $\psi=\phi-2 \varepsilon^{3 / 2} \ln \left(\omega / \omega_{0}\right)$ called shifted phase [40], [14]. The change of $\phi$ into $\psi$ in the higher order terms gives modification to the waveforms, however these are of higher order than $\varepsilon^{3 / 2}$. The double expansion, including the redshift dependence, of $h_{+}$and $h_{\times}$in $\varepsilon$ and $\xi$ leads to the following structure to $\xi$ and $\varepsilon^{3 / 2}$ orders:

$$
\begin{aligned}
h_{+}= & \frac{2 G^{2} m^{2} \varepsilon^{1 / 2} \xi}{c^{4} D r}\left[h_{\times}^{0}+\beta_{1} h_{\times}^{0 \beta}\right. \\
& +\varepsilon^{1 / 2}\left(h_{\times}^{0.5}+\beta_{1} h_{\times}^{0.5 \beta}-2 \xi h_{\times}^{0}\right) \\
& +\varepsilon\left(h_{\times}^{1}-4 \xi h_{\times}^{0.5}\right. \\
& \left.+\beta_{1} h_{\times}^{1 \beta}+h_{\times}^{1 S O}+\beta_{1} h_{\times}^{1 \beta S O}\right) \\
& \left.+\varepsilon^{3 / 2}\left(h_{\times}^{1.5}+h_{\times}^{1.5 S O}+h_{\times}^{1.5 t a i l}\right)\right] .
\end{aligned}
$$

The indices of the various contributions to the waveform refer to the respective order in the double expansion $(\varepsilon, \xi)$, respectively $\beta_{1}=\mathcal{O}(\xi)$. Thus the terms $h_{+}^{0}$ include leading order $\left(\varepsilon^{0}, \xi^{0}\right)$ contributions, while the $h_{\times}^{0 \beta}$ terms are the $\left(\varepsilon^{0}, \beta_{1}\right)$ order contributions. The rest of the terms without explicit spin magnitude dependence are $h_{+}^{0.5}$, of $\left(\varepsilon^{0.5}, \xi^{0}\right)$ orders, $h_{+}^{0.5 \beta}$, of $\left(\varepsilon^{0.5}, \beta_{1}\right)$ orders, $h_{+}^{1}$, of $\left(\varepsilon^{1}, \xi^{0}\right)$ orders, $h_{+}^{1 \beta}$, of $\left(\varepsilon^{1}, \beta_{1}\right)$ orders and $h_{+}^{1.5}$, of $\left(\varepsilon^{1.5}, \xi^{0}\right)$ orders respectively. The leading order contribution including the spin magnitude is $h_{+}^{1 S O}$ of $\left(\varepsilon^{1}, \xi^{0}\right)$ orders, and its corrections $h_{+}^{1 \beta S O}$ and $h_{\times}^{1.5 S O}$ of $\left(\varepsilon^{1}, \beta_{1}\right)$ and $\left(\varepsilon^{1.5}, \xi^{0}\right)$ orders, respectively. The leading order tail term is given by $h_{+}^{1.5 t a i l}$, which is of $\left(\varepsilon^{1.5}, \xi^{0}\right)$ orders. All these explicit expressions are given in the Appendix.

\section{THE PHASE OF THE GRAVITATIONAL WAVEFORM IN THE SPIN DOMINATED REGIME}

The radiative evolution of the orbital angular velocity up to $2 \mathrm{PN}$ orders, including spin-orbit and spin-spin effects was given in [6]. Later, this was complemented by self-spin, quadrupole-monopole ${ }^{4}$ and magnetic dipolemagnetic dipole contributions in [38]. Higher order spin contributions were discussed in Refs. 18], 19].

In order to employ these results for the SDWaveform, we need to derive the $\omega(\varepsilon)$ dependence to $\varepsilon^{2}$ orders accuracy.

Eq. (3) of Ref. [38] (leaving out the magnetic dipolemagnetic dipole contributions, which are only important for magnetar binaries) gives:

$$
\begin{aligned}
\omega= & \frac{\varepsilon^{3 / 2} c^{3}}{G m}\left\{1+\frac{3}{2} \varepsilon+\left(-\frac{\xi}{2}+\chi_{1} \cos \kappa_{1}\right) \varepsilon^{3 / 2}\right. \\
& \left.\times\left[\frac{171}{8}-\chi_{1}^{2}\left(-\frac{3}{8}+\frac{9}{8} \cos ^{2} \kappa_{1}\right)\right] \varepsilon^{2}\right\}
\end{aligned}
$$

Note that the spin-spin terms are shifted to $\varepsilon^{5 / 2} \xi$ order, thus are neglected.

The radiative orbital angular velocity evolution $(\dot{\omega})$ is given by Eq. (7) of Ref. [38]. This evolution for unequal mass case at linear accuracy in $\xi$ and $\varepsilon^{2}$ (with the neglection of $\varepsilon^{2} \xi$ ) becomes

$$
\begin{aligned}
\dot{\omega}= & \frac{96}{5} \frac{\varepsilon^{6} \xi c^{6}}{(G m)^{2}}\left\{1+\frac{1105}{336} \varepsilon\right. \\
& +\left(4 \pi-\frac{79}{12} \xi-\frac{23}{4} \chi_{1} \cos \kappa_{1}\right) \varepsilon^{3 / 2} \\
& +\left[\frac{697465}{9072}\right. \\
& \left.\left.+\chi_{1}^{2}\left(\frac{325}{96} \sin ^{2} \kappa_{1}-\frac{35}{16}\right)\right] \varepsilon^{2}\right\} .
\end{aligned}
$$

Integrating Eq. (7) of Ref. [38] and employing Eq. (26) we find to linear accuracy in $\xi$ and to $\varepsilon^{2}$ orders beyond leading order:

$$
\begin{aligned}
\tau= & \frac{\varepsilon^{-4}}{256}\left\{1-\frac{265}{252} \varepsilon\right. \\
& +\left[5 \xi-\frac{32}{5} \pi+\frac{62}{5} \chi_{1} \cos \kappa_{1}\right] \varepsilon^{3 / 2} \\
& +\left[-\frac{24804463}{508032}\right. \\
& \left.\left.+\chi_{1}^{2}\left(\frac{63}{8}-\frac{577}{48} \sin ^{2} \kappa_{1}\right)\right] \varepsilon^{2}\right\} .
\end{aligned}
$$

Here $\tau$ is a dimensionless time variable defined as

$$
\tau=\frac{c^{3}}{5 G m}\left(1-2 \varepsilon^{1 / 2} \xi\right)\left(t_{c}-t\right)
$$

where $t_{c}$ is the phase and the time at the final coalescence. Integrating twice Eq. (7) of Ref. 38] gives the

\footnotetext{
4 The coefficient p_i of Ref. [38] is $p_{i}=-G^{2} / c^{4} \omega \chi_{i}^{2} m_{i}^{2} / m^{2}$, where $\omega=1$ for black holes.
} 
accumulated orbital phase as

$$
\begin{aligned}
\phi_{c}-\phi= & \frac{\varepsilon^{-3}}{32 \xi}\left\{1+2 \varepsilon^{1 / 2} \xi+\frac{1195}{1008} \varepsilon\right. \\
& +\left(-10 \pi+\frac{3925}{504} \xi+\frac{175}{8} \chi_{1} \cos \kappa_{1}\right) \varepsilon^{3 / 2} \\
& +\left[-\frac{21440675}{1016064}\right. \\
& \left.\left.+\chi_{1}^{2}\left(\frac{375}{16}-\frac{3425}{96} \sin ^{2} \kappa_{1}\right)\right] \varepsilon^{2}\right\}
\end{aligned}
$$

where $\phi_{c}$ is the phase at the final coalescence.

\section{CONCLUDING REMARKS}

In this paper we have analyzed spinning compact binary inspiral in the case when one of the binary components has much larger mass than the other one and it spins fast. In such cases the end of the inspiral is characterized by a much larger spin of the dominant binary component as compared to the orbital angular momentum, such that the parameter $\xi=\chi_{1}\left(L_{N} / S_{1}\right) \leq 0.1$. This latter condition selects the mass ratios $1: 30$ and below. Similar considerations lead to the conclusion, that the second spin can be neglected. Therefore, from all available angular momenta of the system, the larger spin dominates. Hence we call the corresponding waveforms spin-dominated waveforms (SDW).

The SDW were given as a double expansion in the PN parameter $\varepsilon$ and the smallness parameter $\xi$. The angle $\beta_{1}$ between the dominant spin and total angular momentum is also small, of order $\xi$ (this is because the larger spin gives the main contribution to the total angular momentum) The usual mass variables employed for gravitational waveforms, the chirp mass $M_{\text {chirp }}=\left(m_{1} m_{2}\right)^{3 / 5} m^{-1 / 5}=$ $m \eta^{3 / 5}$, and the mass ratio $\nu$ relate to the total mass $m$ and $\xi$ as follows:

$$
\begin{gathered}
M_{\text {chirp }}=m\left[\frac{\varepsilon^{1 / 2} \xi}{\left(1+\varepsilon^{1 / 2} \xi\right)^{2}}\right]^{3 / 5} \\
\approx m \varepsilon^{3 / 10} \xi^{3 / 5}\left(1-\frac{6}{5} \varepsilon^{1 / 2} \xi\right), \\
\nu=\varepsilon^{1 / 2} \xi .
\end{gathered}
$$

The limits of validity of these waveforms were studied, their typical lengths in the instrument best sensitivity range were estimated for the Advanced LIGO, the Einstein Telescope, the LAGRANGE and the LISA gravitational wave detectors, respectively. While the SDW hold for any mass ratio smaller than 1 : 30, lower bounds for the mass ratios were derived from the best sensitivity frequency range expected for these forthcoming gravitational wave detectors. The mass ratio lower bounds are
1 : 140 for Advanced LIGO, $7 \times 10^{-4}$ for the Einstein Telescope, $7 \times 10^{-7}$ for the LAGRANGE and $7 \times 10^{-9}$ for the LISA missions, respectively.

The SDW amplitudes were presented to $\left(\varepsilon^{3 / 2}, \xi\right)$ orders; while the phase of the gravitational waves to $\left(\varepsilon^{2}, \xi\right)$ orders (omitting the highest order mixed terms). To this accuracy the amplitude includes the (leading order) spinorbit contributions. The phase includes the (leading order) spin-orbit, the self-spin and the mass quadrupolemass monopole contributions. Note that expressing the mass ratio in terms of $\xi$ shifts the usual $\varepsilon$ order, and this is what happens with the spin-spin contributions, which would appear only at higher orders. This is consistent with the smaller body's spin being insignificant in the chosen parameter range in comparison with the larger spin and orbital angular momentum.

A comparison showed that the derived SDW, when written with the same level of detail as the waveforms given in Appendix A of Ref. [14], are approximately 80\% shorter. This is due to the smaller number of variables and most importantly, the second series expansion we employ. We expect that the SDW will turn useful both in modeling gravitational waves emitted by binary systems consisting of an astrophysical black hole with a smaller black hole or a neutron star companion; and for supermassive black hole encounters.

\section{ACKNOWLEDGEMENTS}

LÁG was partially supported by COST Action MP0905 "Black Holes in a Violent Universe." ZK was supported by OTKA Grant No. 100216. We acknowledge the support of the European Union / European Social Fund Grant TÁMOP-4.2.2.C-11/1/KONV-2012-0010.

\section{Appendix A: Explicit Spin-dominated waveforms}

We give below the various contributions to the waveform expressed in the double expansion $(\varepsilon, \xi)$, remembering that $\beta_{1}=\mathcal{O}(\xi)$. The $\left(\varepsilon^{0}, \xi^{0}\right)$ contributions are

$$
\begin{aligned}
4 h_{+}^{0}= & \sum_{+,-}\left[\left(\sin ^{2} \theta-2\right) \cos \left(2 \phi_{n} \pm 2 \psi\right) c_{1}^{( \pm 0)}\right. \\
& \left.-2 \sin \kappa_{1} \sin 2 \theta \sin \left(\phi_{n} \pm 2 \psi\right) k^{( \pm)}\right] \\
& +6 \sin ^{2} \kappa_{1} \sin ^{2} \theta \cos 2 \psi \\
2 h_{\times}^{0}= & \sum_{+,-}\left[\cos \theta \sin \left(2 \phi_{n} \pm 2 \psi\right) c_{1}^{( \pm 0)}\right. \\
& \left.-2 \sin \theta \sin \kappa_{1} \cos \left(\phi_{n} \pm 2 \psi\right) k^{( \pm)}\right]
\end{aligned}
$$


while the $\left(\varepsilon^{0}, \beta_{1}\right)$ contributions read

$$
\begin{aligned}
2 h_{+}^{0 \beta S O}= & \sum_{+,-}\left[\sin 2 \theta \sin \left(\phi_{n} \pm 2 \psi\right) c_{2}^{( \pm 0)}\right. \\
& \left.+\sin \kappa_{1}\left(\sin ^{2} \theta-2\right) \cos \left(2 \phi_{n} \pm 2 \psi\right) k^{( \pm)}\right] \\
& -3 \sin 2 \kappa_{1} \sin ^{2} \theta \cos 2 \psi \\
h_{\times}^{0 \beta S O}= & \sum_{+,-}\left[\cos \theta \sin \kappa_{1} \sin \left(2 \phi_{n} \pm 2 \psi\right) k^{( \pm)}\right. \\
& \left.+\sin \theta \cos \left(\phi_{n} \pm 2 \psi\right) c_{2}^{( \pm 0)}\right] .
\end{aligned}
$$

The $\left(\varepsilon^{0.5}, \xi^{0}\right)$ corrections to the gravitational waveforms $h_{+}$are

$$
\begin{aligned}
64 h_{+}^{0.5}= & 4 \cos \theta \sin \kappa_{1} \sin ^{2} \theta\left[-45 \sin ^{2} \kappa_{1} \cos 3 \psi\right. \\
& \left.+\cos \psi\left(5 \sin ^{2} \kappa_{1}-4\right)\right] \\
& +\sum_{+,-}\left\{-\sin ^{2} \kappa_{1} \sin \theta\left[\left(\sin ^{2} \theta\right.\right.\right. \\
& -2)\left(\sin \left(3 \phi_{n} \pm \psi\right) k^{( \pm)}\right) \\
& \left.-45\left(2-3 \sin ^{2} \theta\right)\left(\sin \left(\phi_{n} \pm 3 \psi\right) k^{( \pm)}\right)\right] \\
& +\sin \theta\left[\left(\sin \left(\phi_{n} \pm \psi\right) c_{1}^{( \pm 0.5)}\right)\right. \\
& \left.-9\left(\sin { }^{2} \theta-2\right)\left(\sin \left(3 \phi_{n} \pm 3 \psi\right) c_{2}^{( \pm 0.5)}\right)\right] \\
& +2 \cos \theta \sin \kappa_{1}\left[\left(\cos \left(2 \phi_{n} \pm \psi\right) c_{3}^{( \pm 0.5)}\right)+9(2\right. \\
& \left.\left.\left.-3 \sin ^{2} \theta\right)\left(\cos \left(2 \phi_{n} \pm 3 \psi\right) c_{1}^{( \pm 0)}\right)\right]\right\}, \quad(\mathrm{A} 5
\end{aligned}
$$

\begin{tabular}{|c|c|c|}
\hline$n$ & $i$ & $a_{(i)}^{( \pm n)}$ \\
\hline \multirow[t]{2}{*}{0} & 1 & $\mp 2 k^{( \pm)}$ \\
\hline & 2 & $\mp k^{( \pm)}$ \\
\hline \multirow[t]{11}{*}{0.5} & 1 & $4 k^{( \pm)}\left(6-\sin ^{2} \theta\right)$ \\
\hline & 2 & $4 k^{( \pm)}$ \\
\hline & 3 & $\pm 2\left(6-\sin ^{2} \theta\right) k^{( \pm)}$ \\
\hline & 4 & $12 k^{( \pm)}$ \\
\hline & 5 & $\pm 2 k^{( \pm)}\left(2 \sin ^{2} \theta-3\right)$ \\
\hline & 6 & $-2 k^{( \pm)}$ \\
\hline & 7 & $\mp 2 c_{2}^{( \pm 0)}\left(6-\sin ^{2} \theta\right)$ \\
\hline & 8 & $\pm 2 k^{( \pm)}$ \\
\hline & 9 & $44-34 \sin ^{2} \theta \pm 2\left(5 \sin ^{2} \theta-46\right) \cos \kappa_{1}$ \\
\hline & 10 & $22 \pm 46 \cos \kappa_{1}$ \\
\hline & 11 & $-2 k^{( \pm)}\left(3-2 \sin ^{2} \theta\right)$ \\
\hline \multirow[t]{15}{*}{1} & 1 & $\pm 8 k^{( \pm)}$ \\
\hline & 2 & $6 k^{( \pm)}\left(\sin ^{2} \theta+5\right)$ \\
\hline & 3 & $2 k^{( \pm)}\left(4-\sin ^{2} \theta\right)$ \\
\hline & 4 & $\pm 2 k^{( \pm)}\left(2 \sin ^{4} \theta+11 \sin ^{2} \theta-38\right)$ \\
\hline & 5 & $6 k^{( \pm)}\left(3 \sin ^{2} \theta+5\right)$ \\
\hline & 6 & $\pm 2 k^{( \pm)}\left(4 \sin ^{2} \theta+19\right)$ \\
\hline & 7 & $-2 k^{( \pm)}\left(3 \sin ^{2} \theta-4\right)$ \\
\hline & 8 & $\pm 2 k^{( \pm)}\left(4-\sin ^{2} \theta\right)$ \\
\hline & 9 & $\pm 6 k^{( \pm)}\left(5+\sin ^{2} \theta\right)$ \\
\hline & 10 & $\mp 4 k^{( \pm)}$ \\
\hline & 11 & $k^{( \pm)}\left(22+29 \sin ^{2} \theta-16 \sin ^{4} \theta\right)$ \\
\hline & 12 & $2 k^{( \pm)}$ \\
\hline & 13 & $\pm 6 k^{( \pm)}\left(3 \sin ^{2} \theta+5\right)$ \\
\hline & 14 & $-k^{( \pm)}\left(20 \sin ^{2} \theta+11\right)$ \\
\hline & 15 & $\mp 2 k^{( \pm)}\left(3 \sin ^{2} \theta-4\right)$ \\
\hline \multirow[t]{17}{*}{1.5} & 1 & $\pm 4 k^{( \pm)}\left(\sin ^{2} \theta-6\right)$ \\
\hline & 2 & $\pm 4 k^{( \pm)}\left(\sin ^{4} \theta+42 \sin ^{2} \theta-166\right)$ \\
\hline & 3 & $16 k^{( \pm)}$ \\
\hline & 4 & $8 k^{( \pm)}\left(\sin ^{4} \theta+8 \sin ^{2} \theta-28\right)$ \\
\hline & 5 & $8 k^{( \pm)}\left(-332+94 \sin ^{2} \theta+\sin ^{4} \theta\right)$ \\
\hline & 6 & $\pm 8 k^{( \pm)}\left(38-42 \sin ^{2} \theta-9 \sin ^{4} \theta\right)$ \\
\hline & 7 & $-16 k^{( \pm)}\left(152-46 \sin ^{2} \theta-9 \sin ^{4} \theta\right)$ \\
\hline & 8 & $\pm 24 k^{( \pm)}\left(3 \sin ^{2} \theta-10\right)$ \\
\hline & 9 & $-8 k^{( \pm)}\left(160-204 \sin ^{2} \theta-63 \sin ^{4} \theta\right)$ \\
\hline & 10 & $\pm 4 k^{( \pm)}\left(3-2 \sin ^{2} \theta\right)$ \\
\hline & 11 & $-8 k^{( \pm)}\left(14+3 \sin ^{2} \theta\right)$ \\
\hline & 12 & $-16 k^{( \pm)}\left(15 \sin ^{2} \theta+76\right)$ \\
\hline & 13 & $-8 k^{( \pm)}\left(5 \sin ^{2} \theta+166\right)$ \\
\hline & 14 & $-8 k^{( \pm)}\left(80+63 \sin ^{2} \theta\right)$ \\
\hline & 15 & $\pm 4 k^{( \pm)}\left(166-125 \sin ^{2} \theta-8 \sin ^{4} \theta\right)$ \\
\hline & 16 & $\mp 8 k^{( \pm)}\left(38-61 \sin ^{2} \theta-24 \sin ^{4} \theta\right)$ \\
\hline & 17 & $\pm 8 k^{( \pm)}\left(5-4 \sin ^{2} \theta\right)$ \\
\hline
\end{tabular}

$$
\begin{aligned}
32 h_{\times}^{0.5}= & -16 \sin 2 \kappa_{1} \sin \psi \sin ^{2} \theta \\
& +\sum_{+,-}\left\{\operatorname { c o s } \theta \operatorname { s i n } ^ { 2 } \kappa _ { 1 } \operatorname { s i n } \theta \left[\cos \left(3 \phi_{n} \pm \psi\right) k^{( \pm)}\right.\right. \\
& \left.+45\left(\cos \left(\phi_{n} \pm 3 \psi\right) k^{( \pm)}\right)\right] \\
+ & \frac{1}{2} \sin 2 \theta\left[\left(\cos \left(\phi_{n} \pm \psi\right) c_{4}^{( \pm 0.5)}\right)\right. \\
+ & \left.9\left(\cos \left(3 \phi_{n} \pm 3 \psi\right) c_{2}^{( \pm 0.5)}\right)\right] \\
+ & 2 \sin \kappa_{1}\left[\left(\sin \left(2 \phi_{n} \pm \psi\right) c_{5}^{( \pm 0.5)}\right)\right. \\
& \left.\left.-9 \cos 2 \theta\left(\sin \left(2 \phi_{n} \pm 3 \psi\right) c_{1}^{( \pm 0)}\right)\right]\right\}
\end{aligned}
$$

TABLE I: The terms $a_{i}^{( \pm n)}$ in all PN orders, $n$ is the PN order, $i$ is the number of the constant in the appropriate PN order.

while the $\left(\varepsilon^{0.5}, \beta_{1}\right)$ contributions read 
TABLE II: The terms $b_{(i) 0}^{( \pm n)}$ in all PN orders, $n$ is the PN order, $i$ is the number of the constant in the appropriate PN order.
TABLE III: The terms $d_{(i) 0}^{( \pm n)}$ in $0.5,1$ and $1.5 \mathrm{PN}$ orders, $n$ is the $\mathrm{PN}$ order, $i$ is the number of the constant in the appropriate $\mathrm{PN}$ order.

\begin{tabular}{|c|c|c|}
\hline$n$ & $i$ & $d_{(i) 0}^{( \pm n)}$ \\
\hline \multirow[t]{11}{*}{0.5} & 1 & $5\left(3 \sin ^{2} \theta-2\right)$ \\
\hline & 2 & -1 \\
\hline & 3 & - \\
\hline & 4 & -5 \\
\hline & 5 & - \\
\hline & 6 & 3 \\
\hline & 7 & $-3\left(2-3 \sin ^{2} \theta\right)$ \\
\hline & 8 & - \\
\hline & 9 & - \\
\hline & 10 & - \\
\hline & 11 & $3 \cos 2 \theta$ \\
\hline \multirow[t]{15}{*}{1} & 1 & $\mp 4$ \\
\hline & 2 & $6-14 \sin ^{2} \theta$ \\
\hline & 3 & $2\left(\sin ^{2} \theta-1\right)$ \\
\hline & 4 & $\mp 2\left(8-20 \sin ^{2} \theta+7 \sin ^{4} \theta\right)$ \\
\hline & 5 & $6-7 \sin ^{2} \theta$ \\
\hline & 6 & $\mp 16 \sin ^{2} \theta \pm 8$ \\
\hline & 7 & $3 \sin ^{2} \theta-2$ \\
\hline & 8 & $\pm 9\left(\sin ^{2} \theta-2\right)$ \\
\hline & 9 & $\pm 3\left(18-7 \sin ^{2} \theta\right)$ \\
\hline & 10 & \pm 9 \\
\hline & 11 & $4\left(2-8 \sin ^{2} \theta+7 \sin ^{4} \theta\right)$ \\
\hline & 12 & -2 \\
\hline & 13 & $\pm 6\left(9-13 \sin ^{2} \theta\right)$ \\
\hline & 14 & $2\left(7 \sin ^{2} \theta-2\right)$ \\
\hline & 15 & $\mp 18 \cos ^{2} \theta$ \\
\hline \multirow[t]{17}{*}{1.5} & 1 & - \\
\hline & 2 & $\mp 6 \sin ^{4} \theta \pm 72 \sin ^{2} \theta \mp 20$ \\
\hline & 3 & -12 \\
\hline & 4 & $-15 \sin ^{4} \theta+22 \sin ^{2} \theta-8$ \\
\hline & 5 & $1920-2832 \sin ^{2} \theta-84 \sin ^{4} \theta$ \\
\hline & 6 & $\pm 6\left(10-44 \sin ^{2} \theta+27 \sin ^{4} \theta\right)$ \\
\hline & 7 & $-4\left(88-422 \sin ^{2} \theta+171 \sin ^{4} \theta\right)$ \\
\hline & 8 & $\pm 12\left(14-9 \sin ^{2} \theta\right)$ \\
\hline & 9 & $-9\left(28-126 \sin ^{2} \theta+105 \sin ^{4} \theta\right)$ \\
\hline & 10 & - \\
\hline & 11 & $9 \sin ^{2} \theta-4$ \\
\hline & 12 & $-176+756 \sin ^{2} \theta$ \\
\hline & 13 & $12\left(7 \sin ^{2} \theta+80\right)$ \\
\hline & 14 & $-126+189 \sin ^{2} \theta$ \\
\hline & 15 & $\pm 2\left(10-41 \sin ^{2} \theta+36 \sin ^{4} \theta\right)$ \\
\hline & 16 & $\mp 6\left(10-49 \sin ^{2} \theta+44 \sin ^{4} \theta\right)$ \\
\hline & 17 & $\mp 4\left(7-8 \sin ^{2} \theta\right)$ \\
\hline
\end{tabular}


TABLE IV: The terms $b_{(i) 1}^{( \pm n)}$ in 1 and $1.5 \mathrm{PN}$ orders, $n$ is the $\mathrm{PN}$ order, $i$ is the number of the constant in the appropriate $\mathrm{PN}$ order.

\begin{tabular}{|c|c|c|}
\hline$n$ & $i$ & $b_{(i) 1}^{( \pm n)}$ \\
\hline \multirow[t]{15}{*}{1} & 1 & -1 \\
\hline & 2 & - \\
\hline & 3 & - \\
\hline & 4 & $-2\left(2-8 \sin ^{2} \theta+7 \sin ^{4} \theta\right)$ \\
\hline & 5 & - \\
\hline & 6 & $2-7 \sin ^{2} \theta$ \\
\hline & 7 & - \\
\hline & 8 & $-8 \cos ^{2} \theta$ \\
\hline & 9 & $8\left(3-7 \sin ^{2} \theta\right)$ \\
\hline & 10 & 4 \\
\hline & 11 & - \\
\hline & 12 & - \\
\hline & 13 & $-4\left(7 \sin ^{2} \theta-6\right)$ \\
\hline & 14 & - \\
\hline & 15 & $-4\left(2-3 \sin ^{2} \theta\right)$ \\
\hline \multirow[t]{17}{*}{1.5} & 1 & - \\
\hline & 2 & $-15 \sin ^{4} \theta+12 \sin ^{2} \theta-2$ \\
\hline & 3 & $\mp 5$ \\
\hline & 4 & - \\
\hline & 5 & $\mp\left(236-294 \sin ^{2} \theta+21 \sin ^{4} \theta\right)$ \\
\hline & 6 & $3\left(6-36 \sin ^{2} \theta+45 \sin ^{4} \theta\right)$ \\
\hline & 7 & $\mp 3\left(232-510 \sin ^{2} \theta+243 \sin ^{4} \theta\right)$ \\
\hline & 8 & $9\left(6-5 \sin ^{2} \theta\right)$ \\
\hline & 9 & - \\
\hline & 10 & - \\
\hline & 11 & - \\
\hline & 12 & $\mp 348 \pm 591 \sin ^{2} \theta$ \\
\hline & 13 & $\pm\left(273 \sin ^{2} \theta-118\right)$ \\
\hline & 14 & - \\
\hline & 15 & $\left(2-13 \sin ^{2} \theta+12 \sin ^{4} \theta\right)$ \\
\hline & 16 & $-9\left(2-13 \sin ^{2} \theta+12 \sin ^{4} \theta\right)$ \\
\hline & 17 & $-3\left(3-4 \sin ^{2} \theta\right)$ \\
\hline
\end{tabular}

TABLE V: The terms $d_{(i) 1}^{( \pm n)}$ in $1.5 \mathrm{PN}$ order, $n$ is the PN order, $i$ is the number of the constant in the appropriate PN order.

\begin{tabular}{ccc}
\hline \hline$n$ & $i$ & $d_{(i) 1}^{( \pm n)}$ \\
1.5 & 1 & - \\
2 & - \\
3 & 1 \\
4 & - \\
5 & $28-126 \sin ^{2} \theta+105 \sin ^{4} \theta$ \\
6 & - \\
7 & 27 \\
8 & $\left.-22 \sin ^{2} \theta+15 \sin ^{4} \theta\right)$ \\
9 & - \\
10 & - \\
11 & - \\
12 & $-243 \sin ^{2} \theta+108$ \\
13 & $7\left(2-3 \sin ^{2} \theta\right)$ \\
14 & - \\
15 & - \\
16 & - \\
17 & \\
\hline \hline
\end{tabular}

$$
\begin{aligned}
64 h_{+}^{0.5 \beta}= & 4 \cos \kappa_{1} \cos \theta \sin ^{2} \theta\left[135 \cos (3 \psi) \sin ^{2} \kappa_{1}\right. \\
& \left.+\cos \psi\left(4-15 \sin ^{2} \kappa_{1}\right)\right]+\sum_{+,-}\{2 \cos \theta[9(2 \\
& \left.-3 \sin ^{2} \theta\right)\left(\cos \left(2 \phi_{n} \pm 3 \psi\right) c_{6}^{( \pm 0.5)}\right) \\
& \left.+\cos \left(2 \phi_{n} \pm \psi\right) c_{7}^{( \pm 0.5)}\right]-\sin \kappa_{1} \sin \theta\left(\sin ^{2} \theta\right. \\
& -2)\left[27\left(\sin \left(3 \phi_{n} \pm 3 \psi\right) c_{1}^{( \pm 0)}\right)\right. \\
& \left.+\sin \left(3 \phi_{n} \pm \psi\right) c_{8}^{( \pm 0.5)}\right]+\sin \kappa_{1} \sin \theta[45(2 \\
& \left.-3 \sin { }^{2} \theta\right)\left(\sin \left(\phi_{n} \pm 3 \psi\right) c_{8}^{( \pm 0.5)}\right) \\
& \left.\left.+\sin \left(\phi_{n} \pm \psi\right) c_{9}^{( \pm 0.5)}\right]\right\}
\end{aligned}
$$

$$
\begin{aligned}
32 h_{\times}^{0.5 \beta}= & 32 \sin \psi \sin ^{2} \theta \cos 2 \kappa_{1} \\
& +\sum_{+,-}\left\{\operatorname { c o s } \theta \operatorname { s i n } \kappa _ { 1 } \operatorname { s i n } \theta \left[27 \cos \left(3 \phi_{n} \pm 3 \psi\right) c_{1}^{( \pm 0)}\right.\right. \\
+ & {\left[\cos \left(3 \phi_{n} \pm \psi\right)+45 \cos \left(\phi_{n} \pm 3 \psi\right)\right] c_{8}^{( \pm 0.5)} } \\
& \left.+c_{10}^{( \pm 0.5)} \cos \left(\phi_{n} \pm \psi\right)\right] \\
& -\left[18 \cos 2 \theta\left(\sin \left(2 \phi_{n} \pm 3 \psi\right) c_{6}^{ \pm}\right)\right. \\
& \left.\left.-2 \sin \left(2 \phi_{n} \pm \psi\right) c_{11}^{( \pm 0.5)}\right]\right\} .
\end{aligned}
$$

The $\left(\varepsilon^{1}, \xi^{0}\right)$ corrections to the gravitational waveforms $h_{+}$are 


$$
\begin{aligned}
48 h_{+}^{1}= & 2 \sin ^{2} \theta \sin ^{2} \kappa_{1}\left[\operatorname { s i n } ^ { 2 } \kappa _ { 1 } 5 \left(7 \sin ^{2} \theta\right.\right. \\
& -6)(\cos 2 \psi-4 \cos 4 \psi) \\
& \left.-2\left(15 \sin ^{2} \theta+51\right) \cos 2 \psi\right] \\
& +\sum\left\{1 6 \operatorname { s i n } ^ { 3 } \kappa _ { 1 } \operatorname { s i n } 2 \theta \left(7 \sin ^{2} \theta\right.\right. \\
& +,--\sin \left(\phi_{n} \pm 4 \psi\right) k^{( \pm)}-\left(\sin ^{2} \theta\right. \\
& -3) \sin \left(4 \phi_{n} \pm 2 \psi\right) c_{1}^{( \pm 0)} \\
& -2) \sin ^{2} \kappa_{1} \sin ^{2} \theta \cos \sin ^{2} \theta \cos \left(4 \phi_{n} \pm 4 \psi\right) c_{1}^{( \pm 1)} \\
& +4\left(\sin ^{2} \theta-2\right) \sin _{2}^{( \pm 1)} \\
& +2 \sin \kappa_{1} \sin 2 \theta\left[\sin \left(\phi_{n} \pm 2 \psi\right)\right. \\
& +\sin \left(3 \phi_{n} \pm 2 \psi\right) c_{3}^{( \pm 1)} \\
& \left.-8 \cos { }^{2} \theta \sin \left(3 \phi_{n} \pm 4 \psi\right) c_{2}^{( \pm 0.5)}\right] \\
& +2 \cos \left(2 \phi_{n} \pm 2 \psi\right) c_{4}^{( \pm 1)} \\
& -16 \sin ^{2} \kappa_{1}\left[7 \sin ^{4} \theta-2\left(4 \sin ^{2} \theta\right.\right. \\
& \left.-1)] \cos \left(2 \phi_{n} \pm 4 \psi\right) c_{1}^{( \pm 0)}\right\}
\end{aligned}
$$

$$
\begin{aligned}
24 h_{\times}^{1}= & 60 \cos \kappa_{1} \cos \theta \sin ^{2} \kappa_{1} \sin 2 \psi \sin ^{2} \theta \\
& +\sum_{+,-}\left[8 \operatorname { s i n } ^ { 3 } \kappa _ { 1 } \operatorname { s i n } \theta \left(7 \sin ^{2} \theta\right.\right. \\
& -6) \cos \left(\phi_{n} \pm 4 \psi\right) k^{( \pm)} \\
& -\cos \theta \sin ^{2} \kappa_{1} \sin ^{2} \theta \sin \left(4 \phi_{n} \pm 2 \psi\right) c_{1}^{( \pm 0)} \\
& +2 \sin \kappa_{1} \sin \theta \cos \left(\phi_{n} \pm 2 \psi\right) c_{5}^{( \pm 1)} \\
& +4 \cos \theta \sin ^{2} \theta \sin \left(4 \phi_{n} \pm 4 \psi\right) c_{1}^{( \pm 1)} \\
& +2 \cos \theta \sin \left(2 \phi_{n} \pm 2 \psi\right) c_{6}^{( \pm 1)} \\
& -8 \cos \theta \sin ^{2} \kappa_{1}\left(7 \sin ^{2} \theta-2\right) \sin \left(2 \phi_{n} \pm 4 \psi\right) c_{1}^{( \pm 0)} \\
& -8\left(2-3 \sin ^{2} \theta\right) \sin \kappa_{1} \sin \theta \cos \left(3 \phi_{n} \pm 4 \psi\right) c_{2}^{( \pm 0.5)} \\
& \left.+2 \sin \kappa_{1} \sin \theta \cos \left(3 \phi_{n} \pm 2 \psi\right) c_{7}^{( \pm 1)}\right], \quad(\mathrm{A} 10)
\end{aligned}
$$

while the $\left(\varepsilon^{1}, \beta_{1}\right)$ contributions read

$$
\begin{aligned}
& 24 h_{+}^{1 \beta}=\cos \kappa_{1} \sin \kappa_{1} \sin ^{2} \theta\left[4\left(15 \sin ^{2} \theta+51\right) \cos 2 \psi\right. \\
& \left.+20 \sin ^{2} \kappa_{1}\left(7 \sin ^{2} \theta-6\right)(4 \cos 4 \psi-\cos 2 \psi)\right] \\
& +\sum_{+,-}\left\{\sin 2 \theta \sin \left(3 \phi_{n} \pm 2 \psi\right) c_{8}^{( \pm 1)}\right. \\
& +\sin 2 \theta \sin \left(\phi_{n} \pm 2 \psi\right) c_{9}^{( \pm 1)} \\
& +8 \cos ^{2} \theta \sin 2 \theta \sin \left(3 \phi_{n} \pm 4 \psi\right) c_{10}^{( \pm 1)} \\
& +2 \sin \kappa_{1} \cos \left(2 \phi_{n} \pm 2 \psi\right) c_{11}^{( \pm 1)} \\
& +8 \sin ^{2} \kappa_{1} \sin 2 \theta\left(7 \sin ^{2} \theta\right. \\
& -3) \sin \left(\phi_{n} \pm 4 \psi\right)\left[ \pm 3 k^{( \pm)}+4 \sin ^{2} \kappa_{1}\right] \\
& +16 \sin \kappa_{1}\left(2-8 \sin ^{2} \theta\right. \\
& \left.+7 \sin ^{4} \theta\right) \cos \left(2 \phi_{n} \pm 4 \psi\right) c_{12}^{( \pm 1)} \\
& +\sin \kappa_{1} \sin ^{2} \theta\left(\sin ^{2} \theta-2\right)\left[\cos \left(4 \phi_{n} \pm 2 \psi\right) c_{12}^{( \pm 1)}\right. \\
& \left.\left.-8 \cos \left(4 \phi_{n} \pm 4 \psi\right) c_{2}^{( \pm 0.5)}\right]\right\} \text {, } \\
& 12 h_{\times}^{1 \beta}=30 \cos \theta \sin \kappa_{1} \sin 2 \psi \sin ^{2} \theta\left(3 \sin ^{2} \kappa_{1}\right. \\
& -2)+\sum_{+,-}\left\{\sin \theta \cos \left(\phi_{n} \pm 2 \psi\right) c_{13}^{( \pm 1)}\right. \\
& +2 \cos \theta \sin \kappa_{1}\left[\sin \left(2 \phi_{n} \pm 2 \psi\right) c_{14}^{( \pm 1)}\right. \\
& \left.+4\left(7 \sin ^{2} \theta-2\right) \sin \left(2 \phi_{n} \pm 4 \psi\right) c_{12}^{( \pm 1)}\right] \\
& +\cos \theta \sin \kappa_{1} \sin ^{2} \theta\left[\sin \left(4 \phi_{n} \pm 2 \psi\right) c_{12}^{( \pm 1)}\right. \\
& \left.-8 \sin \left(4 \phi_{n} \pm 4 \psi\right) c_{2}^{( \pm 0.5)}\right] \\
& +\sin \theta\left[\cos \left(3 \phi_{n} \pm 2 \psi\right) c_{15}^{( \pm 1)}\right. \\
& \left.+4\left(2-3 \sin ^{2} \theta\right) \cos \left(3 \phi_{n} \pm 4 \psi\right) c_{10}^{( \pm 1)}\right] \\
& +4 \sin ^{2} \kappa_{1} \sin \theta\left(6-7 \sin ^{2} \theta\right)\left(\mp 3 k^{( \pm)}\right. \\
& \left.\left.-4 \sin ^{2} \kappa_{1}\right) \cos \left(\phi_{n} \pm 4 \psi\right)\right\} .
\end{aligned}
$$

The $\left(\varepsilon^{1}, \xi^{0}\right)$ and $\left(\varepsilon^{1}, \xi\right)$ SO corrections to the gravitational waveforms $h_{+}$are

$$
\begin{aligned}
h_{+}^{1 S O}=\frac{\chi_{1}}{2} \sin \theta \sum_{+,-}\left[ \pm k^{( \pm)} \sin \left(\phi_{n} \pm \psi\right)\right], \\
h_{\times}^{1 S O}=\frac{\chi_{1}}{2} \sin \theta\left\{2 \sin \kappa_{1} \sin \psi \sin \theta\right. \\
\left.\quad+\cos \theta \sum_{+,-}\left[ \pm k^{( \pm)} \cos \left(\phi_{n} \pm \psi\right)\right]\right\},
\end{aligned}
$$

$$
\begin{aligned}
\frac{2}{\chi_{1}} h_{+}^{1 \beta S O}= & \sum_{+,-}\left\{\mp k^{ \pm} \cos \theta \sin \left(2 \phi_{n} \pm \psi-\phi_{1}\right)\right. \\
& +\sin \kappa_{1} \sin \theta\left[ \pm \sin \left(\phi_{n} \pm \psi\right)\right. \\
& \left.\left. \pm \cos \left(\phi_{n} \pm \psi-\phi_{1}\right)\right]\right\} .
\end{aligned}
$$




$$
\begin{aligned}
\frac{4}{\chi_{1}} h_{\times}^{1 \beta S O}= & -\left[2\left(\sin \phi_{1}+2\right) \cos \kappa_{1} \sin \psi\right. \\
& \left.+2 \cos \phi_{1} \cos \psi\right] \sin ^{2} \theta \\
& +\sum_{+,-}\left\{2 \operatorname { c o s } \theta \operatorname { s i n } \kappa _ { 1 } \operatorname { s i n } \theta \left[ \pm \cos \left(\phi_{n} \pm \psi\right)\right.\right. \\
& \left.\mp \sin \left(\phi_{n} \pm \psi-\phi_{1}\right)\right] \pm\left(\sin ^{2} \theta\right. \\
& \left.-2) k^{ \pm} \cos \left(2 \phi_{n} \pm \psi-\phi_{1}\right)\right\},
\end{aligned}
$$

The $\left(\varepsilon^{1.5}, \xi^{0}\right)$ corrections to the gravitational waveforms $h_{+}$are

$$
\begin{aligned}
12288 h_{+}^{1.5}= & 12 \cos \theta \sin \kappa_{1} \sin ^{2} \theta\{\cos 3 \psi[1701(2 \\
& \left.-3 \sin ^{2} \theta\right) \sin ^{4} \kappa_{1}+72 \sin ^{2} \kappa_{1}\left(63 \sin ^{2} \theta\right. \\
& +178)]+\cos \psi\left[-14\left(2-3 \sin ^{2} \theta\right) \sin ^{4} \kappa_{1}\right. \\
& \left.-8 \sin ^{2} \kappa_{1}\left(7 \sin ^{2} \theta+162\right)+16\left(\sin ^{2} \theta+66\right)\right] \\
& \left.-4375\left(2-3 \sin ^{2} \theta\right) \sin ^{4} \kappa_{1} \cos 5 \psi\right\}+\sum_{+,-}\left[2 \left(\sin ^{2} \theta\right.\right.
\end{aligned}
$$$$
-2) \sin ^{4} \kappa_{1} \sin ^{3} \theta k^{( \pm)} \sin \left(5 \phi_{n} \pm \psi\right)
$$$$
+4 \cos \theta \sin ^{3} \kappa_{1} \sin ^{2} \theta \cos \left(4 \phi_{n} \pm \psi\right) c_{1}^{( \pm 1.5)}
$$$$
+16 \cos \theta \sin \kappa_{1} \cos \left(2 \phi_{n} \pm \psi\right) c_{2}^{( \pm 1.5)}
$$$$
+1250 \sin ^{4} \kappa_{1} \sin \theta\left(105 \sin ^{4} \theta\right.
$$$$
\left.-126 \sin ^{2} \theta+28\right) k^{( \pm)} \sin \left(\phi_{n} \pm 5 \psi\right)
$$$$
+625\left(\sin ^{2} \theta-2\right) \sin ^{3} \theta \sin \left(5 \phi_{n} \pm 5 \psi\right) c_{3}^{( \pm 1.5)}
$$$$
+6 \sin ^{2} \kappa_{1} \sin \theta \sin \left(3 \phi_{n} \pm \psi\right) c_{4}^{( \pm 1.5)}
$$$$
+243\left(\sin ^{2} \theta-2\right) \sin ^{2} \kappa_{1} \sin ^{3} \theta \sin \left(5 \phi_{n} \pm 3 \psi\right) c_{2}^{( \pm 0.5)}
$$$$
+4 \sin \theta \sin \left(\phi_{n} \pm \psi\right) c_{5}^{( \pm 1.5)}
$$$$
+5000 \cos \theta \sin ^{3} \kappa_{1}\left(15 \sin ^{4} \theta-12 \sin ^{2} \theta\right.
$$$$
+2) \cos \left(2 \phi_{n} \pm 5 \psi\right) c_{1}^{( \pm 0)}
$$$$
-1250 \cos \theta \sin \kappa_{1} \sin ^{2} \theta\left(5 \sin ^{2} \theta\right.
$$$$
-6) \cos \left(4 \phi_{n} \pm 5 \psi\right) c_{1}^{( \pm 1)}
$$$$
+1875 \sin ^{2} \kappa_{1} \sin \theta\left(8-22 \sin ^{2} \theta\right.
$$$$
\left.+15 \sin ^{4} \theta\right) \sin \left(3 \phi_{n} \pm 5 \psi\right) c_{2}^{( \pm 0.5)}
$$$$
+216 \cos \theta \sin \kappa_{1} \cos \left(2 \phi_{n} \pm 3 \psi\right) c_{6}^{( \pm 1.5)}
$$$$
+27 \sin \theta \sin \left(3 \phi_{n} \pm 3 \psi\right) c_{7}^{( \pm 1.5)}
$$$$
+54 \cos \theta \sin \kappa_{1} \sin ^{2} \theta \cos \left(4 \phi_{n} \pm 3 \psi\right) c_{8}^{( \pm 1.5)}
$$$$
\left.+54 \sin ^{2} \kappa_{1} \sin \theta \sin \left(\phi_{n} \pm 3 \psi\right) c_{9}^{( \pm 1.5)}\right] \text {, }
$$

$$
\begin{aligned}
& 6144 h_{\times}^{1.5}=192 \cos \kappa_{1} \sin \kappa_{1} \sin ^{2} \theta[\sin \psi(64 \\
& \left.-\sin ^{2} \kappa_{1}\left(7 \sin ^{2} \theta-6\right)+4 \sin ^{2} \theta\right) \\
& \left.+27 \sin 3 \psi \sin ^{2} \kappa_{1}\left(7 \sin ^{2} \theta-6\right)\right] \\
& +\sum_{+,-}\left\{+4 \sin ^{3} \kappa_{1} \sin ^{2} \theta \sin \left(4 \phi_{n} \pm \psi\right) c_{10}^{( \pm 1.5)}\right. \\
& -2 \cos \theta \sin ^{4} \kappa_{1} \sin ^{3} \theta \cos \left(5 \phi_{n} \pm \psi\right) k^{( \pm)} \\
& -243 \cos \theta \sin ^{2} \kappa_{1} \sin ^{3} \theta \cos \left(5 \phi_{n} \pm 3 \psi\right) c_{2}^{( \pm 0.5)} \\
& -625 \cos \theta \sin ^{3} \theta \cos \left(5 \phi_{n} \pm 5 \psi\right) c_{3}^{( \pm 1.5)} \\
& +3 \sin ^{2} \kappa_{1} \sin 2 \theta \cos \left(3 \phi_{n} \pm \psi\right) c_{11}^{( \pm 1.5)} \\
& +27 \cos \theta \sin \theta \cos \left(3 \phi_{n} \pm 3 \psi\right) c_{12}^{( \pm 1.5)} \\
& +1875 \cos \theta \sin ^{2} \kappa_{1} \sin \theta(4 \\
& \left.-9 \sin ^{2} \theta\right) \cos \left(3 \phi_{n} \pm 5 \psi\right) c_{2}^{( \pm 0.5)} \\
& +2 \sin 2 \theta \cos \left(\phi_{n} \pm \psi\right) c_{13}^{( \pm 1.5)} \\
& +27 \sin ^{2} \kappa_{1} \sin 2 \theta \cos \left(\phi_{n} \pm 3 \psi\right) c_{14}^{( \pm 1.5)} \\
& +8 \sin \kappa_{1} \sin \left(2 \phi_{n} \pm \psi\right) c_{15}^{( \pm 1.5)}+4375(2 \\
& \left.-3 \sin ^{2} \theta\right) \sin ^{4} \kappa_{1} \sin 2 \theta k^{( \pm)} \cos \left(\phi_{n} \pm 5 \psi\right) \\
& +108 \sin \kappa_{1} \sin \left(2 \phi_{n} \pm 3 \psi\right) c_{16}^{( \pm 1.5)} \\
& +162 \sin \kappa_{1} \sin ^{2} \theta \sin \left(4 \phi_{n} \pm 3 \psi\right) c_{17}^{( \pm 1.5)} \\
& -2500 \sin ^{3} \kappa_{1}\left(2-13 \sin ^{2} \theta\right. \\
& \left.+12 \sin ^{4} \theta\right) \sin \left(2 \phi_{n} \pm 5 \psi\right) c_{1}^{( \pm 0)} \\
& -1250 \sin \kappa_{1} \sin ^{2} \theta(3 \\
& \left.\left.-4 \sin ^{2} \theta\right) \sin \left(4 \phi_{n} \pm 5 \psi\right) c_{1}^{( \pm 1)}\right\},
\end{aligned}
$$

The $\left(\varepsilon^{1.5}, \xi^{0}\right)$ SO corrections to the gravitational waveforms $h_{+}$are

$$
\begin{aligned}
\frac{2}{\chi_{1}} h_{+}^{1.5 S O}= & 4 \sin \kappa_{1}\left[\cos \kappa_{1} \sin \kappa_{1} \cos 2 \phi_{n}\right. \\
& -\cos 2 \kappa_{1} \cos \theta \sin \phi_{n} \sin \theta \\
& +\cos \kappa_{1} \sin \kappa_{1} \sin ^{2} \theta\left(6 \sin ^{2} \psi\right. \\
& \left.\left.-2+\sin ^{2} \phi_{n}\right)\right] \\
& +\sum_{5,-}\left\{2 \operatorname { c o s } \theta \operatorname { s i n } \kappa _ { 1 } \operatorname { s i n } \theta \left[\left(\mp 3 k^{( \pm)}\right.\right.\right. \\
& \left.\left.-4 \sin ^{2} \kappa_{1}\right) \sin \left(\phi_{n} \pm 2 \psi\right)\right] \\
& +\cos \left(2 \phi_{n} \pm 2 \psi\right)\left[-2 k^{( \pm)}+\left(2 \cos \kappa_{1}\right.\right. \\
& \left.\left.\mp 3) \sin ^{2} \kappa_{1}\right]\left(\sin ^{2} \theta-2\right)\right\}, \quad(\mathrm{A} 19) \\
\frac{1}{\chi_{1}} h_{\times}^{1.5 S O}= & -2 \cos \phi_{n} \sin \kappa_{1}\left(\sin \theta \cos 2 \kappa_{1}\right. \\
& \left.+\cos \theta \sin 2 \kappa_{1} \sin \phi_{n}\right) \\
& +\sum\left\{\operatorname { c o s } \theta \operatorname { s i n } ( 2 \phi _ { n } \pm 2 \psi ) \left[-2 k^{( \pm)}\right.\right. \\
& +,- \\
& \left.+\left(2 \cos \kappa_{1} \mp 3\right) \sin ^{2} \kappa_{1}\right] \\
& +\sin \kappa_{1} \sin \theta\left[\mp 3 k^{( \pm)}\right. \\
& \left.\left.-4 \sin { }^{2} \kappa_{1}\right] \cos \left(\phi_{n} \pm 2 \psi\right)\right\},
\end{aligned}
$$


The $\left(\varepsilon^{1.5}, \xi^{0}\right)$ tail corrections to the gravitational waveforms $h_{+}$are

$$
\begin{aligned}
\frac{2}{\pi} h_{+}^{1.5 t a i l}= & 6 \sin ^{2} \kappa_{1} \sin ^{2} \theta \cos 2 \psi \\
& +\sum_{+,-}\left[\cos \left(2 \phi_{n} \pm 2 \psi\right) c_{1}^{( \pm 0)}\left(\sin ^{2} \theta-2\right)\right. \\
& \left.-2 k^{( \pm)} \sin \kappa_{1} \sin 2 \theta \sin \left(\phi_{n} \pm 2 \psi\right)\right],(\mathrm{A} 21) \\
\frac{1}{\pi} h_{\times}^{1.5 \text { tail }}= & \sum_{+,-}\left[\cos \theta \sin \left(2 \phi_{n} \pm 2 \psi\right) c_{1}^{( \pm 0)}\right. \\
& \left.\left.-2 \sin \kappa_{1} \sin \theta k^{( \pm)} \cos \left(\phi_{n} \pm 2 \psi\right)\right] \text { (A } 22\right)
\end{aligned}
$$

In the above expressions we have introduced the notations $k^{( \pm)}=\cos \kappa_{1} \mp 1$. The constant coefficients $c_{i}^{( \pm n)}$, also appearing in the various PN orders of $h_{\times}$are structured as

$$
\begin{aligned}
c_{(i)}^{( \pm n)}= & a_{(i)}^{( \pm n)}+\sin ^{2} \kappa_{1} \sum_{j=0}^{1}\left(b_{(i) j}^{( \pm n)}\right. \\
& \left.+d_{(i) j}^{( \pm n)} \cos \kappa_{i}\right) \sin ^{2 j} \kappa_{1} .
\end{aligned}
$$

Here $n$ denotes the PN order of the coefficients and $i$, $j$ are serial numbers. The contributions $a_{(i)}^{( \pm n)}, b_{(i) 0}^{( \pm n)}$, $d_{(i) 0}^{( \pm n)}, b_{(i) 1}^{( \pm n)}$ and $d_{(i) 1}^{( \pm n)}$ are given in the Tables I, II, III, IV and $\mathrm{V}$. Note that in the nonprecessing case $\left(\kappa_{1}=0, \pi\right)$ the contributions $a_{(i)}^{( \pm n)}$ (with $k^{+}=0, k^{-}=2$ ) fully define the coefficients $c_{i}^{( \pm n)}$.
[1] R. Sturani, S. Fischetti, L. Cadonati, G. M. Guidi, J. Healy, D. Shoemaker, A. Viceré, J.Phys.Conf.Ser. 243 012007, (2010) E-print: arXiv:1005.0551; F. Pretorius, Class. Quant. Grav. 22425 (2005); M. Campanelli, C. O. Lousto, Y. Zlochower, Phys. Rev. D 74, 041501 (2006); M. Campanelli, C. O. Lousto, Y. Zlochower, Phys. Rev. D 74, 084023 (2006); J. G. Baker, J. Centrella, D. Choi, M. Koppitz, J. van Meter, Phys. Rev. D 73 104002, (2006).

[2] E. Berti, V. Cardoso, A. O. Starinets, Class. Quantum Grav. 26, 163001 (2009), E-print: arXiv:0905.2975.

[3] B. M. Barker, R. F. O'Connell, Phys. Rev. D 12, 329 (1975).

[4] B. M. Barker. R. F. O'Connell, Gen. Relativ. Gravit. 2, 1428 (1979).

[5] L. E. Kidder, C. M. Will, A. G. Wiseman, Phys. Rev. D 47, R4183 (1993).

[6] L. E. Kidder, Phys. Rev. D 52, 821 (1995).

[7] T. A. Apostolatos, C. Cutler, G. J. Sussman, K. S. Thorne, Phys.Rev. D 49, 6274 (1994).

[8] F. D. Ryan, Phys. Rev. D 53, 3064 (1996); R. Rieth, G. Schäfer, Class. Quantum Grav. 14, 2357 (1997); L. Á. Gergely, Z. Perjés, M. Vasúth, Phys. Rev. D 57, 876 (1998); R. F. O'Connell, Phys. Rev. Letters 93, 081103 (2004); C. M. Will, Phys. Rev. D 71, 084027 (2005); J. Zeng, C. M. Will, Gen. Rel. Grav. 391661 (2007); L. Á. Gergely, Z. I. Perjés, M. Vasúth, Phys. Rev. D 58, 124001(1998).

[9] L. Á. Gergely, P. L. Biermann, Astrophys. J. 697, 1621 (2009).

[10] L. Á. Gergely, P. L. Biermann, L. I. Caramete, Class. Quantum Grav. 27, 194009 (2010).

[11] T. A. Apostolatos, Phys. Rev. D 52, 605 (1995); T. A. Apostolatos, Phys. Rev. D 54, 2438 (1996); H. Wang, C. M. Will, Phys. Rev. D 75, 064017 (2007); J. Majár, Phys. Rev. D 80, 104028 (2009); A. Klein, Ph. Jetzer, Phys. Rev. D 81124001 (2010), E-print: arXiv:1005.2046.

[12] L. Á. Gergely, Phys. Rev. D 61, 024035 (1999).

[13] L. Á. Gergely, Phys. Rev. D 62, 024007 (2000).

[14] K. G. Arun, A. Buonanno, G. Faye, E. Ochsner, Phys.
Rev. D 79, 104023 (2009).

[15] E. Poisson, Phys. Rev. D 57, 5287 (1998).

[16] L. Á. Gergely, Z. Keresztes, Phys. Rev. D 67, 024020 (2003).

[17] E. E. Flanagan, T. Hinderer, Phys. Rev. D 75, 124007 (2007), E-print: arXiv:0704.0389, É. Racine, Phys. Rev. D 78, 044021 (2008).

[18] G. Faye, L. Blanchet, A. Buonanno, Phys. Rev. D 74, 104033 (2006); L. Blanchet, A. Buonanno, G. Faye, Phys. Rev. D 74, 104034 (2006); Erratum-ibid. D 75 049903, (2007); Erratum-ibid. D 81 089901, (2010).

[19] L. Á. Gergely, B. Mikóczi, Phys. Rev. D 79, 064023 (2009); L. Á. Gergely, P. L. Biermann, B. Mikóczi, Z. Keresztes, Class. Quantum Grav. 26, 204006 (2009).

[20] L. Á. Gergely, Phys. Rev. D 81, 084025 (2010).

[21] L. Á. Gergely, Phys. Rev. D 82, 104031 (2010), E-print: arXiv:1005.5330

[22] J. Majár, M. Vasúth, Phys. Rev. D 77104005 (2008).

[23] N. J. Cornish, J. S. Key, Phys. Rev. D 82044028 (2010).

[24] R. A. Porto, A. Ross, I. Z. Rothstein E-print: arXiv:1203.2962 (2012).

[25] J. R. Oppenheimer, G. M. Volkoff, Phys. Rev. 55, 374 (1939).

[26] I. Bombaci, Astron. Astrophys. 305, 871 (1996).

[27] L. Á. Gergely, P. L. Biermann, E-print: arXiv:1208.5251 qr-qc (2012).

[28] the LIGO Scientific Collaboration, the Virgo Collaboration, Phys. Rev. D 85, 082002 (2012).

[29] the LIGO Scientific Collaboration, E-print: arXiv:1201.5999

[30] B. Abadie et al. (LIGO Scientific Collaboration \& Virgo) Phys. Rev. D 83122005 arXiv:1102.3781 [gr-qc]. (2011).

[31] G. M. Harry (for the LIGO Scientific Collaboration) Class. Quantum Grav. 27084006 (2010).

[32] K. G. Arun et al., Class. Quantum Grav. 26094027 (2009).

[33] P. Amaro-Seoane et al. arXiv:1201.3621v1.

[34] J. W. Conklin, et. al., arXiv:1111.5264 (2011).

[35] J. R. Gair, I. Mandel, M. C. Miller, M. Volonteri, Gen. 
Relativ. Gravit. 43485 (2011).

[36] A. Buonanno, Y. Chen, M. Vallisneri, Phys. Rev. D 67 104025 (2003); Erratum-ibid. D 74029904 (2006).

[37] Y. Pan, A. Buonanno, Y. Chen nad M. Vallisneri, Phys. Rev. D 69104017 (2004); Erratum-ibid. D 74029905 (2006).

[38] B. Mikóczi, M. Vasúth, L. Á. Gergely, Phys. Rev. D 71,
124043 (2005).

[39] J. Levin, S. T. McWilliams, H. Contreras, Class. Quant. Grav. 28175001 (2011).

[40] L. Blanchet, B. R. Iyer, C. M. Will, A. G. Wiseman Class.Quant.Grav. 13575 (1996). 